\title{
En marge de l'idéologie : antimasque et grotesque dans le Dr Faustus et La Tempête
}

\section{François Laroque}

Marie-Thérèse Jones-Davies (éd.)

\section{(2) OpenEdition \\ Journals}

\section{Édition électronique}

URL : http://journals.openedition.org/shakespeare/437

DOI : $10.4000 /$ shakespeare.437

ISSN : 2271-6424

\section{Éditeur}

Société Française Shakespeare

\section{Édition imprimée}

Date de publication : 1 novembre 1981

Pagination : 99-114

ISBN : 2-86433-016-4

Référence électronique

François Laroque, «En marge de l'idéologie : antimasque et grotesque dans le Dr Faustus et La Tempête ", Actes des congrès de la Société française Shakespeare [En ligne], 3 | 1981, mis en ligne le 01 novembre 2007, consulté le 22 janvier 2020. URL : http://journals.openedition.org/shakespeare/437 DOI : 10.4000/shakespeare.437 


\title{
THEATRE ET IDEOLOGIES :
} Marlowe, Shakespeare

\author{
DIRECTEUR DE LA PUBLICATION \\ M.T. Jones - Davies
}

JEAN TOUZOT Libraire - Editeur

38 , rue Saint-Sulpice 75278 PARIS CEDEX 061982 
1982 Jean Touzot Libiaire-Editeur, Paris.

Reproduction et traduction mzme partielle interdite.

Tous droits réservts potir lous pays

y compris i'U,R.S.S. et les pays scandinaves

ISBN 2-86433-016-4 


\title{
AVANT-PROPOS
}

L'opposition soulignée par Horace entre éthique et esthétique Aut prodesse volunt aut delectare poetae - que les Elisabéthains aiment à évoquer, pourrait être le lien qui rattache les unes aux autres les communications, rencontres et table ronde de notre troisième congrès (1981) sur le thème : "Théátre et Idéologies: Marlowe, Shakespeare.» Toutefois, le résultat de nos échanges montre un dépassement possible de l'alternative éthique / esthétique, puisqu'il met en valeur l'insertion d'idéologies susceptibles de transformer la fonction même de la littérature.

Les pièces analysées dans le contexte de leur temps révèlent de multiples aspects de la vision du monde et de la place que l'homme $y$ occupe, tels que les projette le théâtre anglais au tournant du XVIe et au début du XVIIe siècle.

Une exploration du langage et de la pensée des deux grands dramaturges aboutit à un commentaire sur les courants philosophiques ou religieux, sur les idées parfois contradictoires et sur la curiosité intellectuelle jamais assouvie qui se font jour à travers la rhétorique de la scène et le jeu dramatique, du Faust de Marlowe à La Tempéte de Shakespeare.

\author{
M.T. jones-Davies
}

Présidente de la Société Française Shakespeare 
SOCIETE FRANCAISE SHAKESPEARE Actes de Congrès.1981

\section{THEATRE ET IDEOLOGIES : Marlowe, Shakespeare}

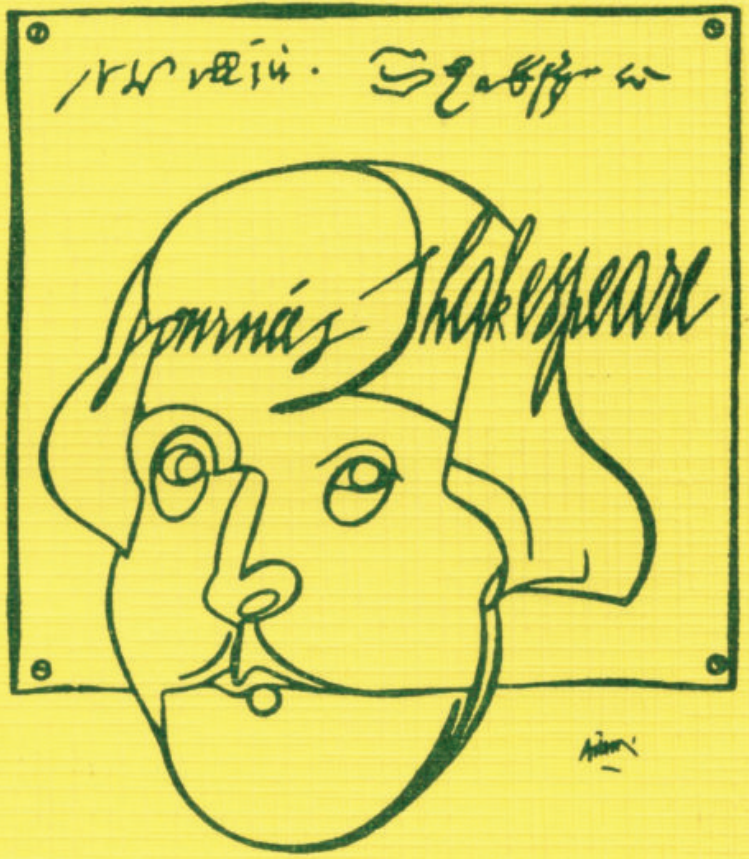

DIRECTEUR DE LA PUBLICATION M.T. Jones - Davies

JEAN TOUZOT Libraire - Editeur 38 , rue Saint-Sulpice 75278 PARIS CEDEX 061982 
Illustration de la couverture : affiche de Valerio Adami, spécialement conçue pour les Journées Shakespeare 1979 au Centre Georges Pompidou 


\section{TABLE DES MATIERES}

M.T. JONES-DAVIES Avant-propos

Théâtre et Idéologies: Marlowe, Shakespeare

S. TRUCHET

The Alchemy of Beauty: aesthetic principles in I Tamburlaine and their relationship to the hermetic ideology.

D. PRUDHOMME

L'idéologie du Juif de Malte et du Marchand de Venise.

D. GOY-BLANQUET

De Hall à Shakespeare : quelques glissements idéologiques opérés par la dramatisation dans Henry $I V$.

M.T. JONES-DAVIES Le Monıde du «si»: Idéologies Incertaines dans Comme il vous Plaira.

G. VENET

Temps et Idéologie : Marlowe, Shakespeare.

J. RICHER

Les Sept Caractères et les Quatre Tempéraments dans Jules César de Shakespeare.

F. LAROQUE

En marge de l'Idéologie : Antimasque et Grotesque dans le Dr Faustus et La Tempête.

A. LECERCLE-SWEET Conscience et Méconnaissance dans Edward The Second de Marlowe.

M.A. CONEJERO Shakespeare's Aesthe tic Scheme. (Extrait).

P.STEWART

Shylock, Shakespeare's Alien.

(Royal Shakespeare Company)

J.RUSSELL BROWN The Romantics' Shakespe are.

R. GILL

The Christian Ideology of Dr Faustus.

J. JACQUOT

Marlowe : de quelques problèmes d'interprétation. 
Rencontres entre Universitaires et Gens de Théâtre

Vendredi 4 décembre 1981
- Stuart Seide :Présentation du

Songe d'une nuit d'Eté. Mise en scène pour le Théâtre de Chaillot janvier 1982

- A propos d'Edouard (II) de

Bernard Turle

Bernard Turle : Métamorphoses de l'auteur et de l'écriture dramatiques. Intervention de Colin Harris (metteur en scène) 


\section{EN MARGE DE L'IDEOLOGIE : ANTIMASQUE ET GROTESQUE DANS LE Dr FAUSTUS ET LA TEMPETE}

Le $D r$ Faustus de Marlowe et La Tempête de Shakespeare sont deux pièces qui valent d'être placées côte à œôte car, bien que séparées d'une vingtaine d'années, elles présentent un certain nombre de parallèlles structuraux, thématiques et linguistiques. Seul, à ma connaissance, M. Marienstras s'en est j'usqu'ici avisé et je ne reviendrai pas sur les tableaux comparatifs qu'il propose en appendice de son étude sur «La littérature élisabéthaine du voyage et $L a$ Tempête» ${ }^{1}$. Je ne m'intéresserai donc ici qu'à ces contrepoints du discours sur la magie, le savoir ou le pouvoir que sont les scènes de farce populaire ou de dérision et qui occupent, dans un cas comme dans l'autre, environ un tiers du texte. Même si les scènes comiques du Dr Faustus ne sont vraisemblablement pas de la main de Marlowe et constituent des ajouts tardifs à la pièce ${ }^{2}$, ce ne serait pas là le premier exemple de collaboration, fût-elle posthume, pour la scène des théâtres publics du Londres élisabéthain, et nous nous en tiendrons, à une variante près, à l'édition de Walter Greg qui suit le texte de 1616. Loin d'être anachroniques ou superflues ces scènes nous paraissent au contraire significatives et novatrices en tant que l'association étrange de la magie savante et du grotesque populaire sera plus tard reprise par Ben Jonson et Shakespeare.

C'est en effet dans la dédicace du Masque of Queenes (1609) que Ben Jonson formule pour la première fois la notion d'antimasque :

[...]Her Majestie [...] had commanded me to think on some dance or show, that might predece hers, and have the place of a foil, or false masque[...] and therefore now devised, that twelve women, in the habit of hags, or witches, sustaining the persons of Ignorance, Suspicion, Credulitie \&c., the opposites to good Fame, should fill that part; not as a masque, but a spectacle of strangeness, producing multiplicity of gesture, and not unaptly sorting with the current, and whole fall of 
the device.

Comme le note Paul Reyher dans sa thèse sur le Masque «si le terme est nouveau, la chose l'est moins» ${ }^{4}$ et les sorcières du Masque of Queenes ont peut-être leur origine dans les danses des Vices et des Sept Péchés Capitaux. Dans ce cas, il convient de considérer que les spectacles infernaux proposés à Faustus par Lucifer à l'acte II et par Mephistopheles à l'acte III sont bel et bien des ancêtres de l'antimasque. Quant à $L a$ Tempête, qui présente un Masque en forme à l'acte IV, il ne fait aucun doute que les scènes d'antimasque y trouvent une place de choix. Mais qu'il s'agisse du Dr Faustus ou de $L a$ Tempête ces mêmes scènes auraient, par leur longueur ou leur variété, suscité la réprobation d'un Francis Bacon qui écrit dans son essai No 37 sur les Masques et les Triomphes :

Let antimasques not be long; they have been commonly of fools, satyrs, baboons, wildmen, antics, beasts, sprites, witches, Ethiopes, pigmies, turquets, nymphs, rustics, Cupids, statuas moving, and the like. As for angels, it is not comical enough to put them in antimasques; and anything that is hideous, as devils, giants, is on the other side as unfit.

Par ce catalogue où, en bon empiriste, Bacon consigne méticuleusement les divers types qui apparaissent dans les antimasques nous sommes déjà sur la piste du grotesque, terme qu'il nous faut à présent tenter de cerner. L'énumération de Bacon suggère que le grotesque, qui est le mode d'être de l'antimasque, surgit du mélange joyeux de créatures balourdes, difformes ou surprenantes. Pour Wolfgang Kayser, auteur d'un ouvrage intitulé The Grotesque in Art and Literature, "[the grotesque] is an attempt to subdue the demonic aspects of the world ${ }^{6}$, définition assez vague mais qui a au moins le mérite de lier le grotesque au fantastique, alors que pour Mikhail Bakhtine

on ne peut arriver à saisir la véritable profondeur, les significations multiples et la force des divers motifs grotesques, que sous l'angle de l'unité de la culture populaire et de la sensation carnavalesque du monde $[.]^{7}$

Dans une étude récente, Neil Rhodes caractérise le grotesque élisabéthain comme procédant du double registre du sermon et de la fête et comme étant marqué par une ambi- 
valence fondamentale :

The Elizabethan grotesque derives from the unstable coalescence of contrary images of the flesh: indulged, abused, purged and damned [...] Frivolity and the macabre, or more generally, laughter and revulsion, are its twin polarities. ${ }^{8}$

J'ai défini ailleurs le grotesque élisabéthain comme un type de discours populaire qui s'inscrivait au cœur d'une triple configuration, celle formée par le calendrier, le corps et la cuisine $^{9}$. Pareil discours, dominé par l'obsession du matériel et du visuel, me paraissait bien illustré par la peinture d'Arcimboldo et de Bruegel l'Ancien. Or, s'il est vrai que dans les exemples du Dr Faustus et de La Tempête les scènes grotesques surviennent dans un arrière-plan de sorcellerie et de magie, elles constituent, par le biais de l'antimasque, une forme de subversion du surnaturel et du fantastique. Il importe donc de les situer dans le contexte festif, carnavalesque, qui est le leur, lequel nous présente la vision d'un corps morcelé, en proie à une incessante métamorphose, avant de nous interroger sur la fonction respective et sur la portée idéologique éventuelle de ces notions dans les deux pièces de Marlowe et de Shakespeare.

En l'absence de tout représentant officiel du Bien et de la Vertu dans le Dr Faustus, c'est le Choeur, qui apparait au début des actes I, III, et IV et qui a le dernier mot de la pièce, qui définit la norme et donne à l'histoire de Faustus valeur d'exemplum à méditer. Dans ces conditions, les scènes de comique populaire et des antimasques proposés par Lucifer et Mephistopheles sont moins une antithèse que l'expression d'une vision populaire des choses qui déforme ou caricature la magie savante à laquelle Faustus décide de s'adonner. Ainsi l'insatiable libido sciendi de Faustus estelle transposée sur le mode burlesque par l'appétit physique de ce pauvre diable qu'est Robin :

$[.$.$] the villain's out of service, and so hungry that$ he would give his soul to the devil for a shoulder of mutton, though it were blood-raw (I. iv. 6-9).

Ce procédé, dont Robert Weimann remarque qu'il est déjà employé dans des Moralités comme Mankind ou King $J_{\text {ohan }}{ }^{10}$, illustre ce que Bakhtine appelle le principe du "rabaissement grotesque» et l'incapacité du clown à décol- 
ler de la pesanteur corperelle et matérielle. Dans la bouche de ce dernier, le monde n'est assimilable qu'après avoir été transformé en nourriture ${ }^{11}$. On trouve dans La Tempête un homologue inverse de ce procédé lorsque, confondant bible et bouteille, Stephano dit à Caliban «Here, kiss the book» (II. ii. 131). Mais cette allusion au "shoulder of mutton" ne doit pas être comprise isolément. Elle se rattache à un système de pensée binaire, qui sévit pendant la fête d'hiver et qui organise en particulier l'espace-temps de la période du carnaval, où les «flesh days» s'opposent aux «fish days» comme le jour à la nuit. Ainsi lorsque Lechery, qui ferme la marche de la parade des Sept Péchés Capitaux, déclare

I am one that loves an inch of raw mutton better than an ell of fried stockfish ...(II. ii. 161-2)

nous sommes mis en présence d'un équivalent dramatique du vieux motif iconographique, illustré par Bruegel entre autres, du Combat de Carnaval et de Carême ${ }^{12}$. Le temps du carnaval, populairement placé sous le signe de l'abondance, plonge dans le temps mythique du Pays de Cocagne. Ainsi Gluttony déclare-t-elle dans l'énoncé d'une généalogie grotesque où les périodes de grand manger et de grand boire, ordinairement disséminées au long du calendrier (St Martin, Carnaval, Mi-Carême), se trouvent miraculeusement réunies:

[..] I come of a royal pedigree : my father was a gammon of bacon, and my mother was a

hogshead of claret wine; my godfathers were these : Peter Pickle-herring and Martin Martlemasbeef. But my godmother was [...] Margery Marchbeer [...] (II. ii. 145-50).

Il est intéressant de noter que si, dans le Faust Book, les chapitres 42 à 44 sont explicitement rattachés à la saison du Carnaval (ils s'intitulent «How Dr. Faustus kept his Shrovetide», "How Dr. Faustus feasted his guests on Ashwednesday" et "How Dr. Faustus the Day following was feasted by the Students, and of his merry Jests with them while he was in their Company»), les allusions directes au calendrier festif s'effacent dans la pièce de Marlowe pour faire place à la succession d'emblèmes vivants. Dans ce rébus parlant, le jeu des acteurs et la dimension visuelle rendent inutile l'indication de régie.

Si l'on en revient à présent au défilé des nourritures grasses dans les tirades de Gluttony et de Lechery, on remar- 
quera que grand boire et grand manger se trouvent réunis et en quelque sorte redoublés grâce au jeu de mot probable sur «hogshead», à la fois tonneau d'une mesure de 54 gallons impériaux et demi et tête de porc. D'autre part, dans le cas de Lechery, on voit les sousentendus obscènes se superposer au registre du culinaire puisque " [the] inch of raw mutton» et " [the] ell of dried stockfish» nous renvoient à la vieille plaisanterie sur les rapports inverses entre la taille et la vitalité phalliques ! L'intempérance sexuelle, qui apparait plus crûment dans le Faust-Book que dans le texte de Marlowe où les personnages féminins sont des ombres (Hélène de Troie) ou des personnages secondaires (la Duchesse de Vanholt, à laquelle Faustus apporte une grappe de raisins en hiver), s'affichait, semble-t-il, plus directement dans le texte de 1604 comme on peut en juger en comparant les deux passages :

1. Texte de 1616 :

Robin What, Dick, look to the horses there till I come again. I have gotten one of Dr. Faustus' conjuring books, and now we'll have such knavery as't passes.

1. Texte de 1604 :

Robin $\mathrm{O}$ this is admirable ! Here I ha' stolen one of Dr. Faustus' conjuring books, and, i'faith, I mean to search some circles for my own use. Now will I make all the maidens in our parish dance at my pleasure stark naked before me, and so by that means I shall see more than e'er I felt or saw yet ${ }^{13}$. En dehors de la satisfaction d'un désir de voyeurisme clairement exprimé, le clown révèle ici son légendaire appétit sexuel par le jeu de mots obscène sur «I mean to search some circles for my own use».

Les critiques se sont étonnés de la satisfaction de Faustus à l'issue de cet antimasque un peu rudimentaire que Weimann rangerait dans ce qu'il appelle «nonrepresentational self-expression ${ }^{14}$. De fait, il semble qu'à l'issue de ce spectacle la curiosité intellectuelle et la soif de savoir du professeur de Wittenberg ne se manifestent pas avec la même ardeur. Le savant athée épris de connaissance se change en épicurien avide de jouissance. Désormais la magie sera plus au service de ses caprices et de ses désirs de facéties que de sa volonté de puissance et de savoir. Sa passion ini- 
tiale pour les secrets de l'univers fait maintenant place à la cosmographie appliquée :

«He now is gone to prove cosmography»

(Choeur, III. 20)

Le deuxieme antimasque ou tableau infernal proposé par Mephistopheles à Faustus dans l'enceinte du Palais du Pape à Rome se donne comme une satire grinçante de la hiérarchie catholique :

... devise what best contents thy mind,

By cunning of thine art to cross the Pope

Or dash the pride of this solemnity,

To make his monks and abbots stand like apes

And point like antics at his triple crown,

To beat about the friars' pates

Or clap huge horns upon the cardinals' heads,

Or any villainy thou canst devise,

And I'll perform it, Faustus. (III. i. 80-8)

Si le Faust-Book se livre à la critique très luthérienne des goinfreries et de la débauche qui sévissaient à la cour papale, l'idée de transformer la solennelle fête de St Pierre en une mascarade grotesque, digne de la fête des Fous ou du Carnaval romain, a peut-être été inspirée à Marlowe par le premier Masque donné à la cour sous le règne d' Elisabeth I lors de la Nuit des Rois de l'année 1559. Le diplomate italien invité à l'occasion de ces réjouissances en donna par lettre le témoignage suivant à Castellani de Mantoue :

As I suppose your Lordship will have heard of the farsa performed in the presence of her Majesty on the day of the Epiphany, and I not having sufficient intellect to interpret it, nor yet the mummery performed after supper on the same day, of crows in the habits of Cardinals, of asses habited as Bishops, and of wolves representing Abbots, I will consign it to silence [.].] Nor will I record the levities and unusual licentiousness practised at the Court in dances and banquets, nor the masquerade of friars in the streets of London ${ }^{15}$.

Antimasque et grotesque sont ici mis au service de la propagande anglicane et de la croisade nationale organisée à l'époque élisabéthaine contre Rome et le Pape. On assiste ici au détournement de la culture comique populaire au service de l'idéologie religieuse et monarchique. 
De même, on peut se demander si la perturbation du banquet par un Faust rendu invisible, idée que Shakespeare reprendra pour la punition des «méchants» dans $L a$ Tempête, n'a pas valeur d'une exclusion symbolique de la communion qui rend ainsi doublement derisoire le rite d'excommunication que le Pape prononce contre l'àme qui ose ainsi troubler son repas. Il y a néanmoins quelque paradoxe à voir ainsi une âme damnée donner des leçons de tempérance au chef spirituel de la chrétienté, à moins que Faustus ne doive déjà être considéré comme l'instrument du diable. C'est là une hypothèse qui n'est nullement invraisemblable si l'on prend en considération la nature des facéties auxquelles Faustus se livre à l'égard de ses victimes et qui ressortissent à la fois au comique carnavalesque et à ce qu'on pourrait appeler le grotesque d'épouvante ${ }^{16}$. Ainsi, les cornes dont Faustus s'amuse à affubler Benvolio et ses acolytes ont-elles une origine multiple : elles renvoient tout d'abord au mythe d'Actéon changé en cerf par Diane, comme le disent explicitement les lignes $51-54$ de la scène 2 de l'acte IV. Le port des cornes se retrouve d'autre part dans les saynètes populaires des «Mummers», qui se produisaient sur la place des villages anglais au moment de Noël, avant de devenir l'emblème ridicule du cocu, comme dans la réplique de Dick à Robin aux lignes 16-18 de la scène iii de l'acte II, ou dans la remarque de Benvolio à Martino à la scène iii de l'acte IV :

Shall I let slip so great an injury

When every servile groom jests at my wrongs

And in their rustic gambols proudly say

Benvolio's head was graced with horns today?

Mais l'obsession des cornes dans cette pièce n'est pas simplement liée à une plaisanterie ou à un vieux rite populaire. Elle est également l'un des symptômespar où se manifeste l'omniprésence du diable, le «dieu cornu» comme l'appelle Margaret Murray dans ses ouvrages sur la sorcellerie 17 Notons que dans la pièce de Greene et Lodge intitulée A Looking Glass for London and England (1590), qui peut avoir influencé Marlowe si l'hypothèse d'une composition tardive (1593) pour le Dr Faustus est la bonne, les deux aspects co-habitent parfaitement dans le bouche d'Adam :

Sir, as I was coming alongst the port-royal of 
Nineveh, there appeared to me a great devil, and as hard-favoured a devil as ever I saw; nay, sir, he was a cuckoldy devil, for he had horns on his head ${ }^{18}$.

Une autre forme de grotesque, plus sinistre celui-là, intervient dans la scène où Faustus, qui porte une fausse tête, se laisse décapiter par Benvolio et Martino. Une fois en possession de la tête coupée, ceux-ci se livrent à des projets de manipulation macabre :

Benv. First, on his head, in quittance of my wrongs, I'll nail huge forked horns and let them hang Within the window where he yoked me first, That all the world may see my just revenge.

Mart. What use shall we put his beard to ?

Benv. We'll sell it to a chimney-sweeper; it will wear out ten birchen brooms, I warrant you.

Mart. What shall his eyes do ?

Benv. We'll pull out his eyes, and they shall serve for buttons to his lips to keep his tongue from catching cold. (IV. iii. 56-64)

A ce plaisir de dissection sadique, qui n'est peutêtre qu'une façon de laisser entrevoir, sur le mode grotesque, la fin horrible de Faustus, on pourrait opposer le travail rassurant et poétique de la métaphore dans le chant d'Ariel, "Those are pearls that were his eyes ...» Mais, lors de la résurrection comique de Faustus qui met en déroute ses ennemis, on se met à songer au scénario de la «Revesby Sword Play» où un personnage appelé Pickle Herring annonce à son père, le «Fool», que ses frères et lui ont décidé de lui couper la tête ${ }^{19}$. On retrouve sur ce point une autre correspondance entre la pièce et les fêtes populaires saisonnières, correspondance voulue par Marlowe puisque, dans le Faust Book, la source de ce passage est fort différente comme on peut en juger par le titre du chapitre 47 : «How four Jugglers cut one anothers Heads off, and set them on again, and Faustus deceived them ${ }^{20}$.

$\mathrm{Si}$ donc les scènes d'antimasque et de grotesque du $\mathrm{Dr}$ Faustus sont reliées entre elles par leur appartenance commune à un comique de type carnavalesque et si on peut les regrouper dans la catégorie de l'utopie populaire du Pays de Cocagne qui marque les rites hivernaux de la «Mummers' Play» et du Carnaval, il semble bien que ces scènes se situent 
en marge de toute idéologie (à l'exception de l'antimasque du palais du Pape).

La pièce de Shakespeare, elle, s'ouvre sur un antimasque qu'il faudrait écrire "ante-masque» avec un $e$, dans la mesure où c'est un avant-masque qui sert de prélude à la pièce $^{21}$, car la tempête vécue à bord du vaisseau royal bouscule la hiérarchie ( What cares these roarers for the name of king ?» (I. i. 16) et célèbre la grand messe du désordre. Le vieux et vertueux conseiller Gonzalo laisse échapper une comparaison obscène tandis que Ferdinand, comme Ariel le rapportera plus tard à son maitre, se croit plongé au milieu de l'enfer : the King's son, Ferdinand,

With hair up-staring, - like reeds, not hair--

Was the first man that leap'd;cried, «Hell is empty,

And all the devils are here ...(I. ii. 212-215)

Cette résurgence des tours de la diablerie et du grotesque d'épouvante fait certes penser à certains passages de la fin du Dr Faustus, mais elle a ici une fonction inverse puisqu'elle est utilisée par un esprit bienfaisant dont le rôle est, comme le dit R. Marienstras, "d'étendre le domaine du bien» ${ }^{22}$. Disons que, dans cette pièce, la diablerie échappe à la représentation et se manifeste essentiellement au niveau du langage et de la métaphore. on la retrouvera surtout dans la violence verbale qui préside au dialogue entre Prospéro, Caliban et Miranda, puisque le vocabulaire utilisé par ces trois personnages appartient au registre de la souffrance ( «cramps», "side-stitches»...), de l'infection («blister, "the red plague»), du difforme («toads», «beetles», «abhorred slave») et d'une sexualité coupable ou perverse («hag-seed», "wicked dam», "to violate», "thy vile race»). Avec l'apparition de Caliban, hybride et homme sauvage s'il en fut, la diablerie originelle dérape vite du côté du monstrueux et l'«ante-masque» devient un "antic masque», c'est-à-dire "a spectacle of strangeness" pour reprendre la définition de Ben Jonson, dans les scènes où les deux bouffons que sont Stephano et Trinculo font équipe avec le "monstre esclave». En un sens, ces trois grotesques correspondent assez bien aux trois dernières allégories du Vice, Sloth, Gluttony et Lechery, dans le défilé des Sept Péchés Capitaux du Dr Faustus. Si enfin l'on s'avise que les trois déesses qui appa- 
raissent dans le Masque de Prospéro, Iris, Junon et Cérès, sont l'antithèse parfaite des trois grotesques, on voit que les scènes comiques de La Tempête ont également la fonction d'un contre-masque qui est là pour faire office de ce que Ben Jonson appelle lui-même un "repoussoir», ou "faux masque», à l'égard des scènes sérieuses de la pièce. Nous verrons plus loin dans quelle mesure ce monde renversé, qui est à la fois l'antagoniste et la caricature de la sphère du Bien, peut jouer un rôle idéologique dans la pièce. Il convient à présent d'interroger ce degré zéro de l'idéologie qu'est le grotesque et qui, grâce à cette extraordinaire création dramatique qu'est Caliban, joue un rôle de premier plan dans La Tempête.

Caliban est un hybride, mi-humain, mi-diabolique, puisqu'il est issu de l'union sexuelle d'une sorcière avec un incube, et il n'a donc pas de place dans l'échelle des êtres de la Renaissance. Comme l'indiquent les figures du bestiaire diabolique qu'il évoque au début de la scène ii de l'acte II («urchin», «apes», «hedgehogs», «adders»), sa rencontre avec Trinculo et Stephano se place sous le signe de la métamorphose : "What have we here ? a man or a fish ?» se demande Trinculo, tandis que Stephano s'exclame : Have we devils here ? Do you put tricks upon's with salvages and men of Ind, ha ?» (58-59). Mais l'avatar le plus surprenant de Caliban est sans doute le monstre à quatre pattes qu'il forme aux yeux de Stephano lorsqu'il se trouve blotti sous sa gabardine avec Trinculo. Il se transforme en quelque gargouille médiévale, en un grylle à deux faces, doté d'une voix de devant ( «forward voice») et d'une voix de derrière («backward voice»). Le monstre devient un diable obscène dont les orifices anaux parlent et disent des horreurs :

... his backward voice is to utter foul speeches and to detract. (II. ii.93-4)

Comme c'était le cas au Moyen Age, et comme nous l'avons vu dans une moindre mesure pour Dr Faustus, les enfers deviennent ici le siège de l'obscénité et du retour du refoulé. Le grotesque obscène atteint son comble lorsque Trinculo sort à reculons de dessous la bâche et que Stephano s'écrie :

Thou art very Trinculo indeed! How cam'st

thou to be the siege of this moon-calt?

can he vent Trinculos? (II. ii. 106-108)

Par le biais d'un langage scatologique («siege» ne signifie-t-il pas excrément et "to vent» expulser par l'anus selon 
I'O.E.D. ?) ${ }^{23}$ ce que décrit ici Stephano n'est ni plus ni moins qu'une scène d'accouchement et de naissance anale. Le grotesque shakespearien rejoint ici le grotesque rabelaisien et la vision carnavalesque analysée par Bakhtine :

Une des tendances principales de l'image grotesque du corps consiste à montrer deux corps dans un seul [...] C'est toujours un corps en état de grossesse ou d'accouchement ${ }^{24}$...

Comme chez Faustus, dont le corps mutilé (tête tranchée et jambe arrachée) repoussait à vue d'oeil devant ses ennemis horrifiés, le corps grotesque de Caliban se multiplie et prolifère sous le regard d'un Stephano qui n'est pas encore assez brouillé par le vin pour qu'il puisse croire à un dédoublement de sa vision. Dès lors qu'il est rendu à une apparence approximativement humaine, ce sont les épithètes les plus changeantes qui vont décliner les différentes identités monstreuses du "veau lunaire»: "shallow monster», "weak monster», "credulous monster», "drunken monster», "puppy-headed monster», "scurvy monster», "poor monster» ... Les sobriquets gouailleurs des ivrognes portent maintenant la métamorphose sur le plan du langage. Le vin qui coule ici à flots, comme dans la révolte de Jack Cade dans 2 Ilenry VI, donne peu à peu à cette scène l'allure d'une fête dissonante, mi-jacquerie, mi-bacchanale, qui va placer les trois grotesques sous l'emprise de la musique d'Ariel. L'effet conjugué de l'ivresse et des rythmes du tambourin semble aussi puissant que les incantations de Faustus et de Mephistopheles puisqu'il aboutit à plonger les trois acolytes dans une attitude de stupeur bestiale :

[...... Then I beat my tabor;

At which, like unback'd colts, they prick'd their ears,

Advanc'd their eyelids, lifted up their noses

As they smelt music ...(IV.i. 175-178)

L'image audacieuse et expressive «they smelt music» rend ici parfaitement par la dégradation de l'auditif dans l'olfactif, le degré d'animalité où sont tombés les grotesques. Par un curieux renversement, c'est Caliban, l'instigateur du complot contre Prospéro, qui s'humanise à présent en mettant en garde ses compagnons contre le danger d'une métamorphose plus hideuse encore : 
And all be turn'd to barnacles, or to apes

With foreheads villainous low ... (IV. i. 247-249)

$\mathrm{Si}$, dans Faustus, le châtiment magique consistait à affubler l'adversaire d'une paire de cornes, comme dans la première partie de la punition d'Actéon par Diane, Prospéro, dans $L a$ Tempête, inflige aux conjurés la deuxième partie de la punition d'Actéon en lâchant à leurs trousses une meute de chiens courants.

Après ces analyses, il faut nous interroger sur la portée de ces scènes qui, pour être comiques, n'en sont pas nécessairement pour autant complètement gratuites. Il est sûr que la conjuration burlesque montée par Caliban contre Prospéro semble justifier a posteriori la sévérité et le pessimisme du maitre à l'égard de son esclave. En un sens Prospéro a besoin de Caliban pour que sa démonstration soit parfaite et il le reconnaîtra d'ailleurs lui-même à la fin de la pièce en déclarant :

............. this thing of darkness I

Acknowledge mine ... (V. i. 275-276)

Caliban est la part d'ombre de Prospéro, il est son «double monstrueux» pourrait-on dire en reprenant l'expression de René Girard ${ }^{25}$. Les scènes de «sabbat»et d'antimasque où les choses fonctionnent à rebours entrent dans une logique des contraires, où le bien est appréhendé à partir de la représentation du mal. Dans une épigraphe datant de 1651, le Jésuite espagnol Balthasar Gracian écrivait :

The things of this world can be truly perceived only by looking at them backwards ${ }^{26}$.

C'est en partant d'une logique analogue, dans son projet de pastorale communiste et utopique, que le vieux Gonzalo expose son rêve d'un retour à l'âge d'or :

I'th' commonwealth I would by contraries

Execute all things ...(II. i. 143-144)

Or, Caliban, l'homme naturel sur qui l'éducation n'a pas de prise, contrarie par son existence même le programme de Gonzalo et en fait une pure chimère. Les scènes d'antimasque et de grotesque de La Tempête ont donc valeur d'un contreexemple et montrent que le mal n'est pas simplement le produit de la civilisation (Alonso, Antonio et Sebastien) mais qu'il existe également au plus bas de l'échelle sociale, là où l'homme est le plus proche de la nature. La fonction idéologi- 
que de ces scènes est donc de subvertir le mythe pastoral selon lequel le mal est du côté de la Cour tandis que le bien se trouve aux côtés des bergers et de la nature. L'antimasque est donc aussi une antipastorale.

Dans le Dr Faustus, les choses sont moins nettement marquées puisque les scènes de comique grotesque constituent moins l'antithèse des scènes sérieuses qu'une sorte de reflet déformé impliquant une chute de l'esprit dans la matérialité corporelle la plus obtuse. Elles ont cependant une valeur d'avertissement en tant qu'elles préfigurent, comme nous l'avons vu, la fin tragique de Faustus. Elles constituent donc de véritables anamorphoses qui, lorsqu'elles sont situées dans la bonne perspective, éclairent le sens de l'oeuvre. Les grotesques jouent dans la pièce un rôle qui ressemble un peu à celui du tableau dans Le Portrait de Dorian Gray : leur pesanteur et leur difformité sont le corrélat objectif des péchés de Faustus. Là aussi l'image du monde renversé sert à faire connaitre les défauts du monde à l'endroit. Mais la justification suprême des scènes comiques de Faustus est que le comique carnavalesque qui s'y déploie est aussi la métaphore de la sorcellerie et de la damnation, comme nous l'avons vu à propos du sens de l'image des cornes qui étaient aussi bien le masque joyeux des «Mummers» que l'attribut sinistre du diable. Des puritains comme Stubbes avaient d'ailleurs, dès 1583 , dénoncé les fêtes populaires saisonnières de l'Angleterre élisabéthaine en expliquant qu'elles n'étaient autres que le rendez-vous de $\operatorname{Satan}^{27}$. Dans un article récent intitulé «Inversion, Misrule and Witchcraft», l'historien anglais Stuart Clark confirme cette analogie lorsqu'il écrit que

$[\ldots$.$] it is certain that what was required of both the$ spectator of festive misrule and the reader of demonological textbooks was formally, and perhaps even substantially, the same $2 . .8$

Ceci ne signifie nullement que Marlowe avait rejoint les rangs des puritains quand il écrivait Faustus mais tout simplement qu'il s'est servi du système d'analogies,mis au point par ces derniers aux fins de leur propagande en guise de contrepoint à l'in trigue principale de sa pièce. Il semble que, dans ce cas particulier, l'idéologie ait été mise au service de l'art. 
Ainsi, dans une pièce comme le Dr Faustus, les scènes d'antimasque et de grotesque nous paraissent bien rester en marge de toute idéologie. Elles servent de contrepoids comique aux élans intellectuels de Faustus et donnent un caractère humain et universel à son itinéraire. Elles ont également une fonction de redoublement et tissent des codes analogiques et anamorphiques qui ont valeur de parallèle structural ou d'avertissement moral.

Dans La Tempête au contraire, pièce fortement imprégnée par le néo-platonisme, les scènes d'antimasque et de grotesque ont, de par la laideur et l'obscénité de leurs protagonistes, valeur d'anti-modèles. Elles sont en quelque sorte les ténèbres extérieures du Bien. Mais, en même temps, par le biais de la logique des contraires, leur existence est nécessaire à la réussite du projet du Bien. Comme le dit Thomas Heywood dans The Hierarchie of the Blessed Angels :

The Divell doth th'Almighty zany. For in those great works which all wonder aske, he is still present with his Anti-maske. ${ }^{29}$

François LAROQUE 


\section{NOTES}

1. Actes du Congrès de Nice de la SAES (1971), pp. 21-49.

2. C'est là I'hypothèse la plus souvent retenue par les critiques. On ne sait en fait rien de précis ou d'incontestable sur le nombre ou le contenu de ces additions à l'in-quarto de 1604, qui peuvent avoir été écrites du vivant de Marlowe par un collaborateur (Rowley ?) ou avoir été payées à un tâcheron par Henslowe en 1602. Il reste que l'essentiel de ces scènes comiques suit de très près le Faust Book, principale source utilisée par Marlowe pour sa pièce.

3. Edition utilisée, Masques and Entertainments by Ben Jonson, Henry Morley ed., London, 1890, pp. 102-3.

4. Les Masques anglais, Etude sur les ballets et la vie de cour en Angle terre (1512-1640), Paris, 1909, p. 171.

5. Edition utilisée, The Essays of Francis Bacon, Everyman, 1906, p. 116.

6. Traduit par Ulrich Weinstein, Bloomington, Indians, 1963, p. 188.

7. L beuvre de François Rabelais et la culture populaire au Moyen Age et sous la Renaissance, traduit par Andrée Robel, Paris, 1970, p. 61.

8. The Elizabethan grotesque, London, 1980 , pp. 4 \& 10

9. «Images et figures du grotesque à l'époque élisabéthaine : calendrier, corps, cuisinew, Cahiers Charles V, 2. Théatre et Société (Avril 1980), pp. 29-39.

10. Shakespeare and the Popular Tradition in the Theatre, Baltimore. 1978, p. 183.

11. «Images et figures du grotesque ..." , ibid, , P, 34 .

12. Voir sur ce point l'article de Claude Gaignebet, «Le combat de Carnaval et de Carême», Annales (1972), pp. 313-345.

13. Cette variante est tirée de l'appendice de l'édition Revels Plays du Dr Faustus, John D. Jump ed., 1962, p. 110.

14. Weimann, op. ctt, p. 83 .

15. Venetian, Papers, VII, p. 11 .

16. Nous proposons cette expression comme équivalent du terme anglais «eldritch" tel qu'il est analysé par Muriel Bradbrook dans son essai intitulé «Marlowe's Doctor Faustus and the Eldritch Tradition» in Essays on Shakespeare and Elizabethan Drama in Honour of Hardin Cratg, Richard Hosley ed., London, 1963.

17. The Witch-cult in Western Europe, Oxford, 1921 et The God of the Witches, Oxford, 1931 (Chapitre 1). 
18. Edition utilisée: Robert Greene, Mermaid Series, Thomas H. Dickinson ed., V, i, p. 148.

19. Chief Pre-Shakespearean Dramas, J.Q. Adams ed., Boston, 1924, The Revesby Sword Play, 11. 135-45, p. 359/2 (2e colonne).

20. The History of the Damnable Life and Deserved Death of John Faustus in Early English Prose Romances, William J. Thoms ed., London, 1889 , p. 866 .

21. Selon Reyher, op. cit. p. 171, c'est là le terme utilisé par Samuel Daniel et Chapman.

22. op. cit., p. 43.

23. «Siege» $3 c$; * to vent» 1 .

24. op. cit., p. 35.

25. La violence et le sacré, Paris, 1972, pp. 200-234.

26. Cité par Stuart Clark in «Inversion, Misrule and the Meaning of Witcheraft», Past and Present $N^{\circ} 87$ (Jan. 81), p. 110.

27. Voir, en particulier, la description que Stubbes donne des «Maygames» dans The Anatomy of Abuses (1583), ed. Furnivall, New Shakespeare Society, London $1877-82$, p. 149.
28. Ibid., p. 104
29. Londres, 1635, p. 415 . 